\title{
Organizing Smart Networks and Humans into Augmented Teams
}

\author{
Martijn Neef, Martin van Rijn, Danielle Keus and Jan-Willem Marck \\ TNO Defence, Security and Safety, The Netherlands \\ \{martijn.neef, martin.vanrijn, danielle.keus, \\ jan_willem.marck\} atno.nl
}

\begin{abstract}
This paper discusses the challenge of turning networks of sensors, computers, agents and humans into hybrid teams that are capable, effective and adaptive. We propose a functional model and illustrate how such a model can be put into practice, and augment the capabilities of the human organization. We specifically focus on the interaction between the human and artificial part of the system, with specific attention to task delegation, role adjustment and adaptive autonomy. In this paper, we introduce the main concepts and report on observations from initial experiments.
\end{abstract}

\section{Introduction}

Networked systems and operations are at the center of many research and development agendas. Our working environments are full of networked devices, and close interactions between humans and networked devices are commonplace. We use sensor networks for remote observations, we interact by means of wireless communication devices, and we benefit from network-accessible information sources. Gradually, we also see networked devices play a more active and cooperative role in operations. We expect this development to continue. Close cooperation between humans and intelligent networks will occur at various levels of cognition, perhaps to the extent that we effectively create 'augmented teams': teams whose capabilities are greatly augmented by the involvement of sensors, networks and artificial actors, and in which technological means practically become part of the team itself. Advances in artificial intelligence fuel such developments, and allow intelligent systems to play a far more pro-active and autonomous role than traditionally. We are already seeing signs of such network-enhanced teams in the military domain, where the availability of networks and smart systems are changing the face of the battlefield. Augmented team concepts will appear in many safety and security domains, because of the incessant need for additional sensing and acting capabilities in such environments.

Despite many research on sensors, networks and intelligent systems, there is little practical work on how to incorporate such systems effectively into human-centered teams. There are two major requirements that we present ourselves with: (a) the approach must be suitable for the current and future state of technology, and (b) the augmented team must exhibit adaptive capabilities. The first requirement is important in view of ongoing developments in system engineering. In most current systems, 
humans are in charge of tasks that demand higher levels of cognition, whereas system components usually take on tasks with lower cognitive requirements. In order to be future-proof, designs must take into account that the traditional division of labour between human and artificial actors will gradually fade away.

A related feature that we seek is adaptivity. Adaptivity refers to the ability of systems to modify its structure or behaviour to obtain a better fit when its circumstances change. There is a lot of interest in adaptive capabilities for systems and organizations, but it is a difficult feature to achieve. Many work on adaptive team concepts stem from the military domain (Klein, 2000), and various examples of adaptive capabilities have been implemented on the battlefield, although mostly in limited form. Most human teams use a fixed role and task allocation. We want our augmented teams to exhibit adaptive capabilities. The team should be able to alter role and task allocations (internal adaptivity) and course of action (external adaptivity) whenever needed. It should be possible to modify positions and activities in a seamless manner. Because an augmented team also includes artificial actors, this implies that it should be possible to have artificial actors switch roles with human actors, and vice versa. Our design concept should cater for such events, and facilitate adaptive behaviours.

\section{A Functional Design for Augmented Teams}

An augmented team consists of a collective of sensors, actuators, information processing systems and humans that are interconnected through an intelligent network. An augmented team has adaptive capabilities with respect to organization structure, role and task allocation and information flow between elements. That implies that roles and tasks may be exchanged between team members without disrupting the integrity of the team and without needing a major redesign of the information flow through the system. It also means that the team can easily accommodate new elements (sensors, actuators, human actors), and that their added capabilities automatically become part of the feature set of the team.

We view an augmented team as a cognitive system, a system that is set in the real world, has perceptive and cognitive capabilities (self-reflection, reasoning, understanding, learning, decision making) and can respond to situations with reason and intention. In conventional automation processes, there is a clear divide between the human team and the technical system. Because of our adaptivity and agility requirements, we intentionally disregard the challenge of proper division of labour between human and artificial team members, and start from a pure functional stance.

We use the Networked Adaptive Interactive Hybrid Systems (NAIHS) model [2, 3] as a blueprint for our hybrid organizations. The NAIHS model describes a typical sensor-data driven networked system from a functional point of view, and considers both human actors and artificial entities to fulfill functional components of the system. NAIHS decomposes systems into functional components, essential functions that need to be fulfilled by actors in the system. The NAIHS model does not prescribe which actor should take responsibility for a certain function, just as long as performance criteria for each function are met during execution. This means that a part of the system could be responsible for fulfilling multiple functions, or that multiple actors could jointly achieve a single function. 


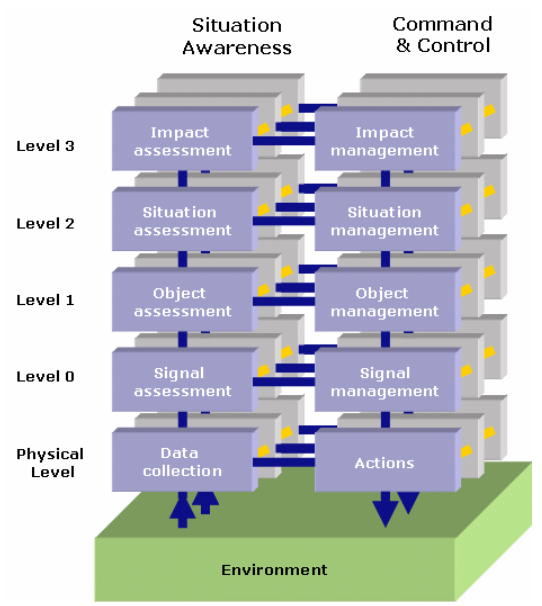

Fig. 1. The Networked Adaptive Interactive Hybrid Systems model [3]

NAIHS uses three principles to decompose a system into functional components: (a) the level of information abstraction, (b) the timescale of desired effects and (c) the physical structure of the system in its environment. For the decomposition in information abstraction level, NAIHS distinguishes between 'situation awareness' components and 'command and control' components. In addition, NAIHS uses four levels of information abstraction, taken from the JDL model [9], ranging from elementary signal assessment and generation (level 0) to high-level situation assessment and planning (level 3). The second dimension, timescale, emphasizes that functions may have different time-constraints. The third dimension, physical structure, captures the physical aspects of each function. NAIHS uses these dimensions as elemental steering guides for the assembly of effective chains of tasks in a networked system. For further details, see Kester [3].

\subsection{Organizing Team Structures}

The functional NAIHS model gives us a transparent way to describe the functions of an augmented team. But how do we organize the teamwork, and describe the various interactions between elements? The dynamic nature of an augmented team makes it unpractical and undesirable to arrange all possible task allocations and interactions in advance. This means that we need a flexible way to describe the tasks of each element, and the relationship it has with other elements. Such descriptions should effectively describe what kind of behaviour one can expect from an actor, and can subsequently be used to arrange effective collaborations between actors.

Actor interaction in networked augmented teams is comparable with interactions in multi-agent systems. We use a specification framework from the agent research community to represent the organization of an augmented team and the interactions between elements. OperA [1] offers a comprehensive methodology and specification language to represent and structure dynamic cooperation of artificial agents. OperA uses three models to represent multi-agent organizations. The Organizational Model 
represents organizational goals and requirements. It describes roles, generic interaction structures, performance criteria, norms, ontologies and other aspects of an organization that define the boundaries of operation. The Social Model represents the agreements that individual elements adopt when they become part of the organization. These 'social contracts' describe tasks associated with a role, obligations, permissions and structural relation with other roles. The Interaction Model represents interaction commitments between elements that specifies the format and frequency of interaction. It can also include agreements on how to solve conflicts and other relevant processes.
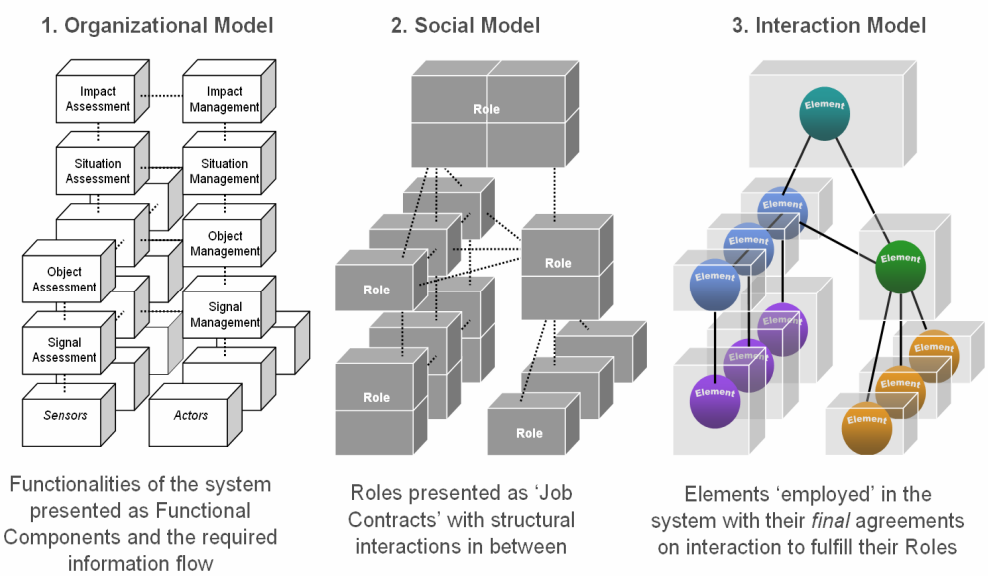

Fig. 2. Using OperA to implement the organizational structure of an augmented team

OperA uses a formal description language to represent contracts, so that the organization can be validated through logical verification methods. Logical verification of the models can reveal unsatisfied objectives or contracts that are not fulfilled. This is, of course, an interesting feature for distributed systems for with respect to task planning and system performance assessment [10].

\subsection{Interaction and Adaptivity}

In an augmented team, the information flow must 'bring' the right (relevant) information to the right functional component. The flow of information is dynamic. Role adoptions at the social level and interaction agreements on the interaction level give rise to flows of information from one actor to another. This means that actors need to make sure that they receive information from other actors in the right form, and at the right time. They learn their information needs when they accept a 'job contract' during role adoption, and ensure that they receive information by negotiating interaction agreements with other actors.

Social and interaction contracts are normally not present in an explicit form in organizations. Human teamwork is bound by common agreements that are usually informal in nature. In an augmented team however, we cannot depend on informal understandings, since artificial entities need to comprehend agreements in order to 
participate. The presence of artificial team members in augmented team makes it necessary to make every collaborative agreement explicit and accessible. This includes agreements between humans. If human actors reach an interaction agreement, their interaction contract must be available in the organization so it can be administered and monitored by other actors. This means that either the actors themselves need to publish the details of the contract, or have a third party capture the details of the agreement.

To facilitate the contract processes, it is wise to create a contract manager. A contract manager is a component that is responsible for maintaining an overview of all elements and their contracts. Upon entering an augmented team, an element needs to accept the interactions contracts that are associated with the roles it will fulfill. The contract manager manages this process and keeps an administration of all contracts. Because of its administrative role, the contract manager is also in position to identify which element fulfils which functionality, and can signal mismatches and impossibilities in task allocation. The contract manager could interact with a process manager to assess the performance of the augmented team. When performance is sub-par, the process manager could instigate adaptive measures by modifying contracts or roles in the organization. The OperA models give us a clear way to describe three types of adaptive organizational behaviour: (1) Interaction adaptivity, in which elements adapt their way of interaction, (2) Role adaptivity, in which actors change roles, and (3) Behavioural adaptivity. The first has the least impact on the structure of the organization, because it only affects the interaction between actors. The last has the most impact, because it affects the fundamental aspects of the organization.

Given the all the above, the essential design tactic of an augmented team becomes as follows. From the business model derive a suitable functional model using the NAIHS structure. Create roles and role descriptions, and design suitable control structures that can administer role adoption for all sorts of actors (interface-mediated role adoption for human actors, protocol-driven role adoption for artificial actors). Develop interaction templates that suit the type of actors in the organization, and design control structures that can help to implement and manage these interactions (e.g. communication, information and collaboration settings). And, to facilitate adaptive behaviours, create behaviour patterns that can help to quickly modify a team's behaviour, as a driver for adaptivity (e.g. define working modes for the entire team). Brought together in a network- and service-oriented framework, these elements would form the basis for the development of augmented teams.

\section{Experiments}

We are currently carrying out experiments to validate the augmented team concept. From a technical perspective, we test the concept on its ability to self-organize workand system structure under changing conditions. From an operational point of view, we want to learn about the cognitive engineering implications of the augmented team concept. Practically, our aim is to demonstrate that it is possible to assemble humans, sensors, actuators, information systems and communication means into an agile and adaptive system that can operative effectively in safety critical environments. 


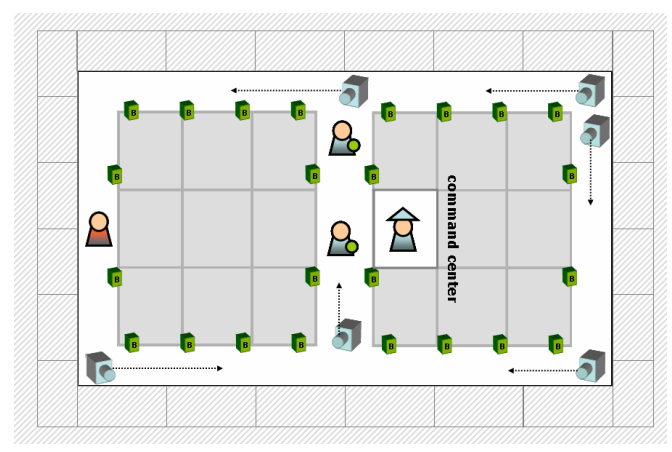

Fig. 3. Schematic layout of the experiment environment

Our experiments take place in an indoor field lab. This lab contains a heterogeneous sensor suite, advanced tracking, tracing and prediction software and a command center with communication and information display facilities. The field lab is set in an actual office environment (the offices of TNO in The Hague) and covers an entire floor. The various sensors and software applications are organized by a serviceoriented architecture and structured following NAIHS principles [2, 3]. The current sensor suite contains cameras and radio beacons, which have been placed along the corridors of the fieldlab. The cameras are used for observation purposes and can recognize objects that have been trained in advance. The radio beacon network is, in conjunction with wearable tags, used as an tracking and tracing system. Information from the cameras and radio tags is combined in a tracker application that integrates sensor information over time and is capable of predicting the trajectory of people when they are out of reach of sensors. This information is displayed in the command center, from where a central coordinator plans actions among the various actors in the environment. For communication purposes, the players have mobile phones at their disposal.

The current set of experiments uses a simple intruder detection and apprehension scenario in which an office safety team needs to figure out the position of an intruder and capture him by inclusion. The team consists of two roaming guards and a coordinator in the command center. The coordinator has access to the information in the network (positions of explorers and predicted location of intruder) and is in charge of directing the explorers. The security team has two main tasks (a) first find the intruder using a search strategy and (b) arrest him by means of enclosure by guards. The security team will have no trouble apprehending the intruder, but the time it takes depends on successful communication and planning. Successful runs ended in less than two minutes, while problematic runs took more than six minutes.

Figure 5 shows which actors play which role in the organization. Artificial actors do not perform any decision making and coordination tasks yet. This condition limits adaptivity in our scenarios to human role change at the moment. Our main scenario involves the transfer of the coordinator role from the player in the control room to one of the mobile guards. This role change is useful when a guard obtains visual contact with the intruder, and has a better view on the situation than the coordinator. 


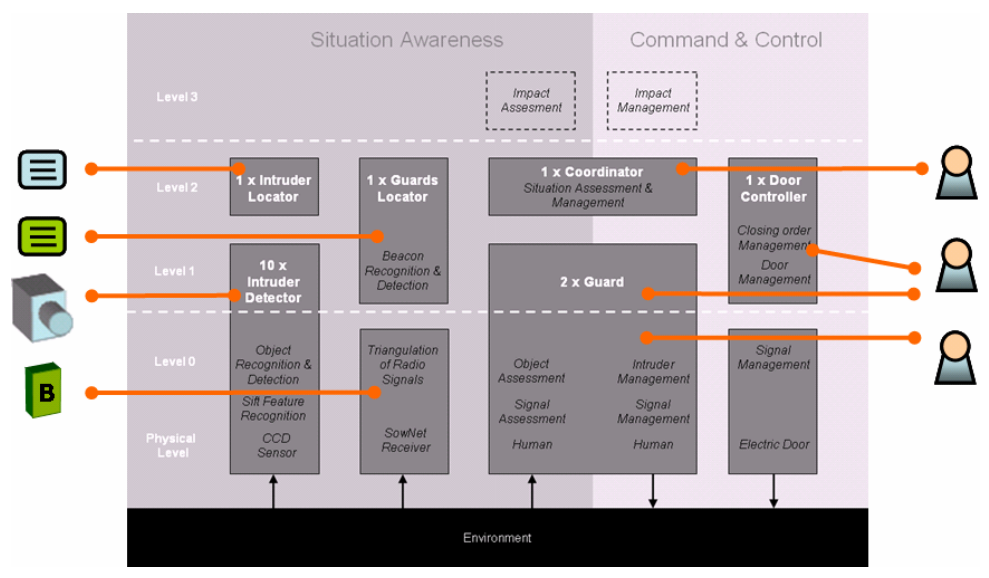

Fig. 4. The basic role layout of the scenario, and the actors that fulfill the various roles. On the left, from top to bottom, the tracker application, the positioning application, the smart camera network and the radio beacon network.

The coordinator-role-change scenario involves a modification of the Social Model. The Organizational Model does not change, because function-wise the organization remains intact. The Interaction Model, the third stage in the OperA suite, does change after role change. The interactions that the original coordinator had with information services and other actors, need to be modified to reflect that there is a new coordinator. In the coordinator-role-change scenario, we use job contracts, predefined packages of roles and interactions to speed up the negotiations phases. A job contract describes (1) designated tasks (e.g. observe, coordinate, inform), (2) task conditions (e.g. area, time-span, network constraints), (3) interaction contracts with actors that fulfill crucial data and information needs, (4) interaction contracts with actors that fulfill an identical role (e.g. to synchronize actions), (5) interaction contracts with actors that depend on the role taker to fulfill crucial data and information needs. For the coordinator role, the job contract package establishes the collaboration with the guards (what do they need to know, how can they be addressed), with the information systems (what information does the coordinator need, and which role provides that information?) and with other systems that are available (what control relations does the coordinator have, and how can they be put into effect?). The players are given instructions on the job contracts in advance, so that they can negotiate collaborations before the scenario starts. This means that, as soon as role change occurs, every actor knows exactly what rules to abide.

At the start of the scenario, the player in the control room accepts the coordinator role, and implements his job contract. He is given the proper information from the information systems (the screens switch on in the control room), and he gives instructions to his guards. The guards have no access to any other information than what the coordinator relays. When the camera system picks up detections of the intruder the coordinator direct the guards to the right hallways in order to enclose the intruder. As soon as a guard has a visual contact on the intruder, then roles change. Since the guard has the best view on the situation, he takes over the coordinator role. 
The coordinator job contract calls for information about the position of actors and intruder detections. In the command room, this information is readily available on screen. The new mobile coordinator needs this information as well, and thus needs to negotiate new interaction contracts with the original providers of the information (the tracking and tracing applications). If the coordinator has a digital information device at his disposal, then he can arrange for that information to appear on his personal device. If he lacks such a device, then his only means to obtain information about the global situation is the actor in the command room. In that situation, the coordinator could opt to set an interaction contract with the command room actor, so that he functions as an information relay. This requires strict instructions from the coordinator on how to convey information, because it is hard to convey complex spatial information through a speech channel. We have tried numerous interaction variants, and strict adherence to communication agreements and proper preparation appears to be critical.

It appears that agile role re-allocation is feasible, as long as the augmented team as a whole takes care of adjusting interaction contracts and information needs. If that succeeds, then adaptive role allocation becomes a powerful feature that paves the way for interesting options, such as the coordinator role-change from our scenario. Other interesting options would be to use human actors as instant sensors and effectors, or have system actors act as substitutes for human actors in case of emergencies.

\section{Design Concerns}

The dynamic deployment of roles among human and artificial introduces many challenges with respect to human - machine teaming. We briefly discuss some apparent design concerns that we encountered in our practical work.

\section{Define who is responsible for roles and task allocation}

Role and task allocation could be a joint responsibility of all entities, or the sole responsibility of an allocation manager. It needs to be clear who is in charge, because directly affects the operational chain of command. In a distributed, adaptive setting, the chain of command may become obfuscated because of changing roles and responsibilities [4], which may cause confusion and loss of coordination.

\section{Ensure transparency of responsibilities and attributes}

Attributes and responsibilities of elements, both artificial and human, should be transparent and observable. This is an essential condition to enable dynamic role deployment. In a regular organization or system, it is clear from the start who or which system is responsible for which task. In a dynamic setting, assignments of elements change, and there is a distinct danger of loss of organization awareness. We advocate the use of explicit social contracts to represent responsibilities and capabilities, and the creation of an administrative role to keep an overview of all elements and their contracts in an organization. This adheres to the notion of observability and directability, which are fundamental principles from cognitive system engineering [4, 8].

\section{Make the type of adaptivity a design choice}

There are many forms of adaptivity. An important decision that needs to be made during design is whether to use prearranged behavioral patterns for adaptive behaviour, or to have adaptivity emerge from an internal collaborative process. To prevent 
'clumsy automation' issues [8] and uncontrollable adaptivity, use as much predefined adaptive measures as possible. For example, work from Parasuraman [7] could be used to pre-define collaboration types between actors, instead of relying on emergent adaptivity.

\section{Prevent communication and interaction issues after role change}

All kinds of communication issues may occur when heterogeneous groups of entities collaborate. These problems are commonly known as interoperability problems. Before two elements can collaborate, there must be an agreement on how to communicate, and through which means. If the elements communicate at different levels, they will most likely fail to reach an agreement. As a rule of thumb, we suggest that elements should only communicate with neighboring elements, on the same level either of abstraction, or directly above or below (see the NAIHS model).

\section{Prevent issues caused by multi-level or multi-role allocation of an element}

It is possible for elements to take on multiple roles or tasks with different characteristics, such as a different levels of abstraction or a different timescale. For each role change, it needs to be checked whether an element is not faced with roles that are too divergent, and will cause performance issues. For instance, a complex information analysis task might not fare well with an immediate physical task that would send the actor into the field. There need to be criteria available to assess the combination of multiple roles, and which can be assessed during contract negotiation.

There are numerous other issues that need to be addressed when dealing with adaptive systems, such as maintaining system awareness, skill degradation concerns and user acceptance. Many are well-known and described in numerous papers about adaptive system design (e.g. [6]).

\section{Conclusions}

This paper discussed the challenge of turning networks of sensors, computers, agents and humans into hybrid teams that are capable, effective and adaptive. We propose a functional model and illustrate how such a model can be put into practice and augment the capabilities of a human organization. We specifically focus on the interaction between the human and artificial parts of the system, with specific attention to task delegation and role adjustment. We use a functional model, the Networked Adaptive Interactive Hybrid Systems (NAIHS) model, as a blueprint for our organizations. The NAIHS model considers both man and machine to fulfill functional roles. To explicate the interactions between these roles, we make use of OperA, an organization modeling framework from multi-agent systems research. These models make it possible to express various aspects of a multi-agent organization, and as a result, help to organize a collection of autonomous agents into a coherent system. Despite of the obvious differences between human and artificial actors, we find that these models form an interesting basis to build hybrid organizations. The use of contracts facilitates the interaction and role transfer between actors, and gives a practical solution to articulate teamwork requirements in augmented teams. We believe that these interaction contracts are essential to fulfill basic cognitive engineering needs such as mutual 
observability, directability and resilience, and help to achieve complex features challenges such as organizational scaling, restructuring and agility.

\section{References}

1. Dignum, V., Dignum, F., Meyer, J.-J.C.: An Agent-Mediated Approach to the Support of Knowledge Sharing in Organizations. Knowledge Engineering Review 19(2), 147-174 (2004)

2. Kester, L.J.H.M.: Model for Networked Adaptive Interactive Hybrid Systems. In: Proceedings of COGIS 2006: COGnitive systems with Interactive Sensors, Paris (2006)

3. Kester, L.J.H.M.: Designing Networked Adaptive Interactive Hybrid Systems. In: Proceedings of the IEEE International Conference on Multisensor Fusion and Integration for Intelligent Systems 2008 (MFI 2008), Seoul, Republic of Korea, pp. 516-521 (2008)

4. Klein, G., Pierce, L.G.: Adaptive teams. In: Proceedings of the 6th International Command \& Control Research \& Technology Symposium (ICCRTS 2001), Annapolis, MD, USA (2001)

5. Klein, G., Woods, D.D., Bradshaw, J.M., Hoffman, R.R., Feltovich, P.J.: Ten Challenges for Making Automation a "Team Player" in Joint Human-Agent Activity. IEEE Intelligent Systems 19(6), 91-95 (2004)

6. Miller, C.A., Funk, H., Goldman, R., Meisner, J., Wu, P.: Implications of Adaptive vs. Adaptable UIs on Decision Making: Why "Automated Adaptiveness" is Not Always the Right Answer. In: Proceedings of the 1st International Conference on Augmented Cognition, Las Vegas, NV, USA (2005)

7. Parasuraman, R., Sheridan, T.B., Wickens, C.D.: A model for types and levels of human interaction with automation. IEEE Transactions on Systems, Man, and Cybernetics, Part A 30(3), 286-297 (2000)

8. Sarter, N.B., Woods, D.D., Billings, C.E.: Automation Surprises. In: Salvendy, G. (ed.) Handbook of Human Factors and Ergonomics, 2nd edn., pp. 1926-1943. Wiley, New York (1997)

9. Steinberg, A.N., Bowman, C.L., White, F.E.: Revisions to the JDL Data Fusion Model. In: Proceedings of the SPIE: Sensor Fusion: Architectures, Algorithms, and Applications III, vol. 3719, pp. 430-441 (1999)

10. van der Vecht, B., Dignum, F., Meyer, J.-J.C., Neef, M.: A Dynamic Coordination Mechanism Using Adjustable Autonomy. In: Sichman, J.S., Padget, J., Ossowski, S., Noriega, P. (eds.) COIN 2007. LNCS, vol. 4870, pp. 83-96. Springer, Heidelberg (2008)

11. Woods: Human-centered software agents: Lessons from clumsy automation. In: Flanagan, J., Huang, T., Jones, P., Kasif, S. (eds.) Human centered systems: Information, Interactivity, and Intelligence, National Science Foundation, Washington, DC, pp. 288-293 (1997) 